\title{
Editorial
}

\section{The Applications of Metamaterials}

\author{
Hui Feng Ma, ${ }^{1}$ Sanming $\mathrm{Hu}^{2,3}$ Yun Gui Ma, ${ }^{4}$ Yun Lai, $^{5}$ and Karu Esselle ${ }^{6}$ \\ ${ }^{1}$ State Key Laboratory of Millimeter Waves, Southeast University, Nanjing 210096, China \\ ${ }^{2}$ School of Engineering and Physical Sciences, Heriot-Watt University, Edinburgh EH14 4AS, UK \\ ${ }^{3}$ Institute of Microwave Techniques, University of Ulm, $89069 \mathrm{Ulm}$, Germany \\ ${ }^{4}$ State Key Lab of Modern Optical Instrumentation, Centre for Optical and Electromagnetic Research, \\ Department of Optical Engineering, Zhejiang University, Hangzhou 310058, China \\ ${ }^{5}$ School of Physical Science and Technology, Soochow University, Suzhou 215006, China \\ ${ }^{6}$ Department of Engineering, Macquarie University, Sydney, NSW 2109, Australia
}

Correspondence should be addressed to Hui Feng Ma; hfma@seu.edu.cn

Received 2 April 2015; Accepted 2 April 2015

Copyright (C) 2015 Hui Feng Ma et al. This is an open access article distributed under the Creative Commons Attribution License, which permits unrestricted use, distribution, and reproduction in any medium, provided the original work is properly cited.

Metamaterials are artificial structures composed of periodic or nonperiodic subwavelength macro cells. The advantages of metamaterials are that their effective medium properties can be controlled by designing the macro units to constitute special materials that do not exist in nature. Metamaterial is a research area with importantly theoretical significance and challenges, which has achieved significant breakthroughs in theory. However, the lack of applications is a bottleneck to restrict the further development of metamaterial. Hence it is very important to develop and seek the significant applications in this area. The papers selected for this special issue present some potential applications of metamaterial, and we are pleased to share them with the readers.

The special issue contains seven papers, where two papers are related to control of radiation patterns by using metasurface. One paper presents a modified two-dimensional Luneburg lens based on bulky metamaterials. One paper introduces an improved artificial transmission line with low characteristic impedance to minimize the Balun. One paper provides a method to design the temperature sensor by using metamaterial. The other two papers propose the combination of the metamaterial structures with traditional microstrip antennas to achieve performance improvement.

In a paper entitled "Suppression of Specular Reflections by Metasurface with Engineered Nonuniform Distribution of Reflection Phase," X. M. Yang et al. study a novel planar metasurface characterizing nonuniform distribution of reflection phase. A design example of the metasurface is presented. In the example, three-layer stacked square patches with variable sizes are utilized as the reflecting elements of the metasurface. It is verified by both theoretical calculation and numerical simulation that the metasurface is capable of suppressing specular reflections of conducting plates significantly for two orthogonal incident polarizations by scattering most incident energy in off-specular directions. The proposed metasurface is an effective approach of RCSreduction for conducting objects and has the potential of providing broadband performance.

In the paper entitled "Control of the Radiation Patterns Using Homogeneous and Isotropic Impedance Metasurface," F. Yang et al. use a cylindrical metasurface to conduct an antenna system. They point out that the radiation patterns of this antenna can be manipulated by altering the surface impedance of this metasurface. Also, they give theoretical derivations, together with some numerical simulation, to demonstrate the validity of this antenna system. The proposed surface has many good properties, like isotropy, homogeneity, low profile, and high selectivity of frequency.

In the paper entitled "Modified Luneburg Lens Based on Metamaterials," H. Chen et al. present a modified twodimensional Luneburg lens composed of a number of concentric layers, and the varied I-shaped units are used in the 
design. Both simulation and experiment results prove the fact that cylindrical waves can be transformed into plane waves when penetrating through the modified Luneburg lens.

In the paper entitled "Compact Two-Section Half-Wave Balun Based on Planar Artificial Transmission Lines," C. Liu et al. present and analyse an improved artificial transmission line with low characteristic impedance. Then, using seven sections of this transmission line, a compact $900 \mathrm{MHz}$ Balun is designed, fabricated, and experimentally verified to achieve a significant size reduction of around $90 \%$.

In a paper entitled "Design of a Compact and High Sensitivity Temperature Sensor Using Metamaterial," S. Zemouli et al. present a sensor of temperature using the metamaterial which is composed of split resonant rings (SRRs) and a cut wire deposed on the surface of $\mathrm{BaTiO}_{3}$ substrate. The permittivity of $\mathrm{BaTiO}_{3}$ has big dependence with the temperature, which can affect the resonant frequency of the SRRs and cut wire; hence the resonant peak of the structures will be sensitive to the temperature.

In the paper entitled "Metamaterial Inspired Microstrip Antenna Investigations Using Metascreens," M. T. Asim and M. Ahmed introduce a metascreen and apply it under the proposed metamaterial antenna and over a simple patch antenna to investigate their performances. The $-10 \mathrm{~dB}$ bandwidths are $14.56 \%$ and $22.86 \%$ for the metamaterial antenna with single and double metascreens which are observed from measured results, respectively. Similarly, the $-10 \mathrm{~dB}$ bandwidth for the simple patch with inverted metascreen is also measured as $9.6 \%$ and $16.66 \%$ for dual band operation. Hence, the proposed technique has potential applications in UWB wireless.

In a paper entitled "Comparison Analysis of Single Loop Resonator Based Miniaturized Triple-Band Planar Monopole Antennas," K. Wu et al. present four kinds of metamaterial miniaturized triple-band antennas by integrating a single loop resonator (SLR) as part of the radiation patch. Two kinds of SLRs (rectangular and circular shaped radiation patches) are investigated and discussed, which can provide the additional operating frequencies of miniaturized planar monopole antennas. Two feeding techniques including coplanar waveguide and microstrip feeds are also presented, respectively. The numerical and experimental results show good performances of the miniaturized triple-band antennas consisting of a SLR, which have potential applications for wireless communications.

\section{Acknowledgments}

We would like to thank the authors for their excellent contributions and patience in assisting us. Finally, the fundamental work of all the reviewers of these papers is also very warmly acknowledged.

Hui Feng Ma Sanming $\mathrm{Hu}$ Yun Gui Ma

Yun Lai

Karu Esselle 

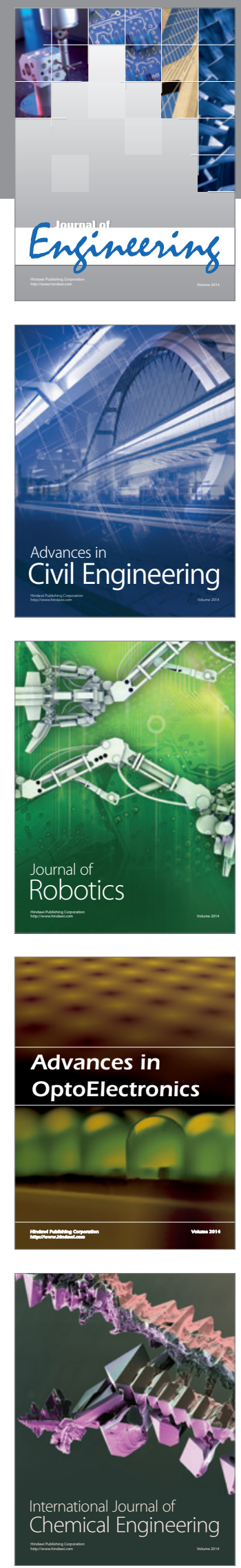

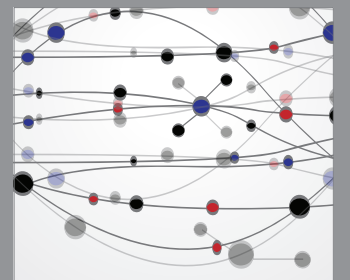

The Scientific World Journal
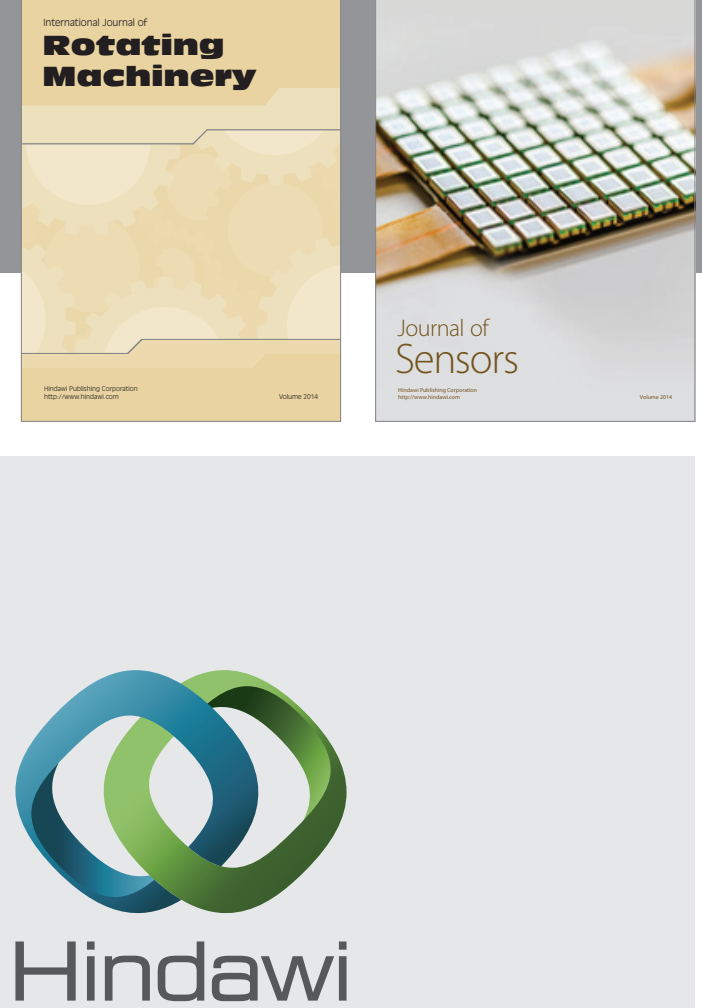

Submit your manuscripts at http://www.hindawi.com
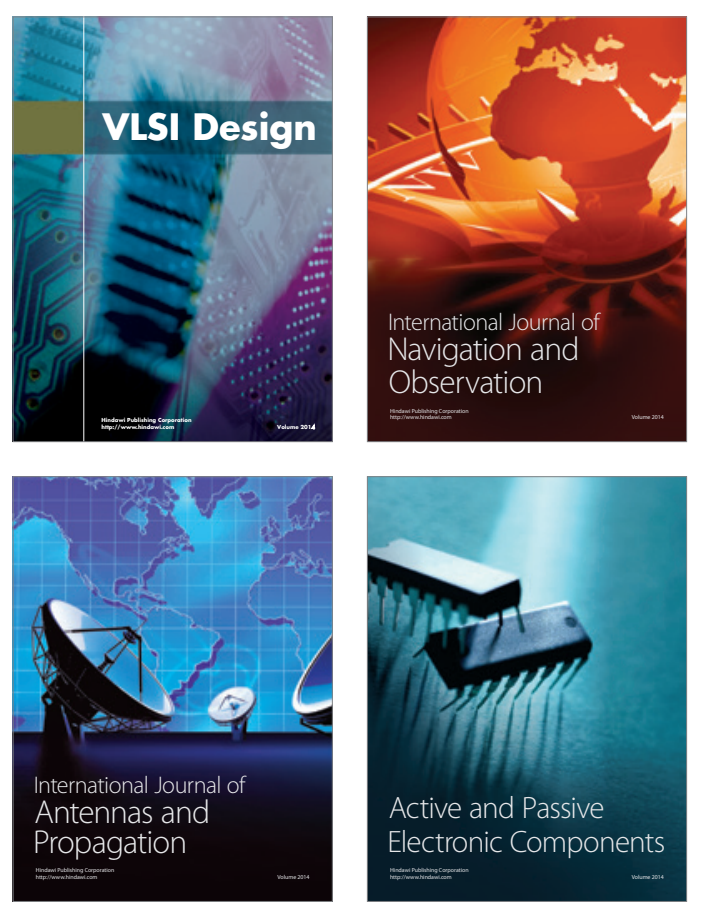
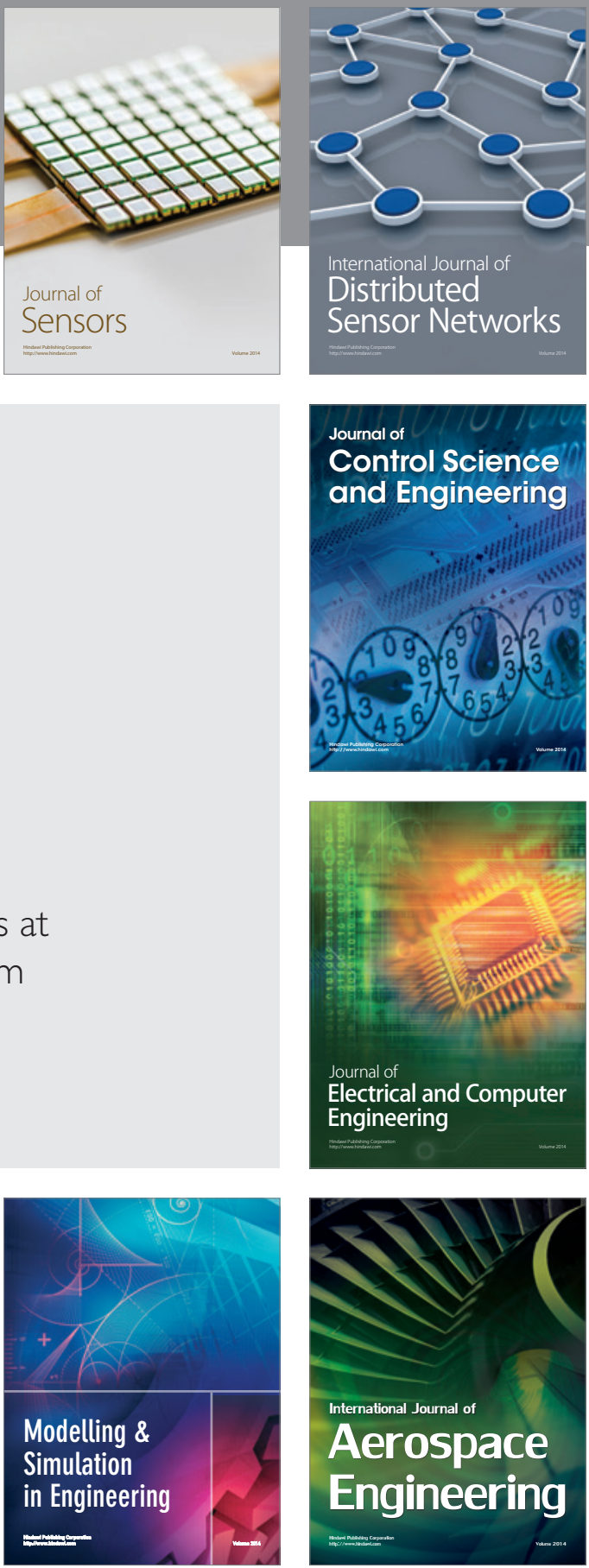

Journal of

Control Science

and Engineering
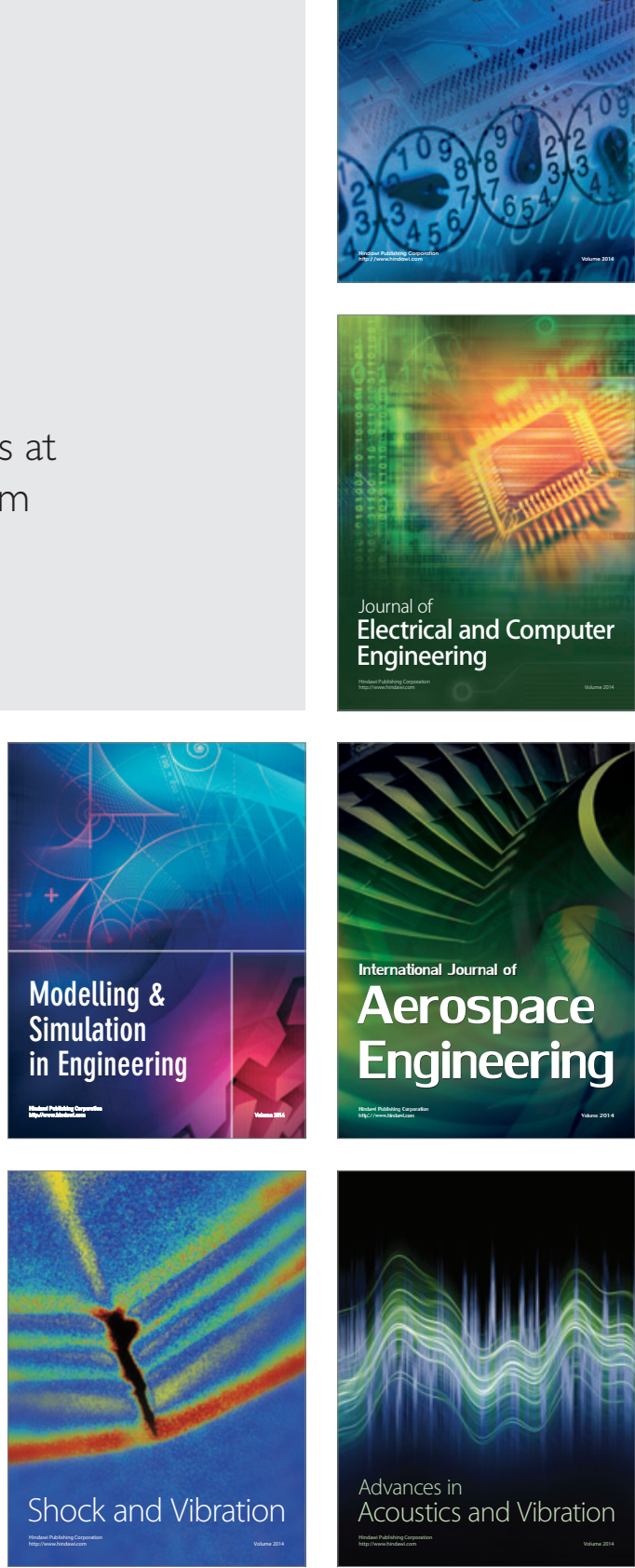